different outcomes exist after HF programme completion based on body mass index (BMI).

Methods A multi-centre retrospective observational study was carried out in 3 hospitals' HF clinics. New patients referred to each HF service over a 12 month period were reviewed, or in a 24 month period in one centre, with a left ventricular ejection fraction (LVEF) $<50 \%$ were eligible for inclusion. Patient records were obtained to collect patient demographics, New York Heart Association (NYHA) class symptoms, NTproBNP, LVEF and medications at initial referral and at programme completion. Hospitalisation rate for $\mathrm{HF}$ and mortality was also noted. Patient characteristics and outcomes were compared between non-obese $(\mathrm{BMI}<29.9)$ and obese (BMI $>30)$.

Results 93 patients were included for analysis and a follow up of (mean $( \pm \mathrm{SD}) \quad 16.5$ months $( \pm 8.8)$ was obtained. 63 $(67.7 \%)$ had a BMI <29.9 and $30(32.3 \%)$ had a BMI $>30$. Obese patients were more likely to be male $(p=0.03)$, diabetic $(p=0.05)$ and have hypertension $(p<0.001)$. No significant differences in medical or device therapy was noted between groups. Obese patients had a greater improvement in their LVEF following programme completion $(10.5 \%$ ( \pm 11.7$)$ versus $7.95 \%( \pm 10.7))$, although this did not reach statistical significance $(p=0.34)$. Obese patients had significantly lower NT-pro BNP levels at HF programme completion ((median(IQR)) (546.4 () versus 962 (), $\mathrm{P}=0.02$ ) however this improvement was not significantly greater than that seen in non-obese patients $(p=0.8)$. Although no difference in mortality was noted between the groups (8 (12.7\%) versus 3 (10\%), $\mathrm{p}=0.73$ ), obese patients had significantly fewer HF hospitalisations than the non-obese group $(0.33( \pm 0.74)$ versus 0.04 $( \pm 0.2), p=0.005)$.

Conclusion Despite higher rates of diabetes and hypertension in obese patients, there was no significant difference in mortality, LVEF improvement and NT-proBNP improvement following HF programme completion between the 2 groups. However, obese patients had significantly less HF hospitalisations during the study period than non-obese patients.

\section{INFECTIVE ENDOCARDITIS POST-TRANSCATHETER AORTIC VALVE IMPLANTATION: EXPERIENCE FROM AN IRISH TERTIARY REFERRAL CENTRE}

${ }^{1} \mathrm{~A}$ Buckley, ${ }^{1} \mathrm{~B}$ Armstrong, ${ }^{1} \mathrm{R}$ Tanner, ${ }^{2} \mathrm{~S}$ Hassan, ${ }^{2} \mathrm{~B}$ Moran, ${ }^{1} \mathrm{~J}$ Byrne, 'S Groarke, ${ }^{2}$ R Margey, ${ }^{1,2}$ I Casserly. 'Mater Misericordiae University Hospital, Dublin, Ireland; ${ }^{2}$ Mater Private Hospital, Dublin, Ireland

\subsection{6/heartjnl-2020-ICS.21}

Background Prior studies of infective endocarditis (IE) posttranscatheter aortic valve implantation (TAVI) have reported an incidence of $1-2 \%$, a median time from TAVI to presentation with IE of $\sim 1$ year, and 1-year mortality rates of 40$50 \%$. In addition, a higher rate of enterococci as the culprit microorganism compared to staphylococci and streptococci has been reported.

Objectives To determine the clinical characteristics, microorganism spectrum, and outcomes of IE post-TAVI in an Irish tertiary referral centre.

Methods Using a prospective TAVI database, all patients diagnosed with IE post-TAVI were identified. Pre-defined baseline demographic, procedural and follow-up data were analysed. Specific additional retrospective analysis of medical records

\begin{tabular}{ll} 
Abstract 21 Table 1 & Culprit Micro-organism \\
\hline Culprit microorganism & $\mathrm{N}(\%)$ \\
\hline Streptococci & $7(41.1)$ \\
Strep mitis & $2(11.7)$ \\
Strep salivarius & $2(11.7)$ \\
Strep infantarius & $1(5.8)$ \\
Strep gordonii & $1(5.8)$ \\
Abiotrophia Defectiva & $1(5.8)$ \\
Enterococcus faecalis & $3(17.6)$ \\
Staphylococci & $3(17.6)$ \\
Staph aureus & $1(5.8)$ \\
Coagulase-negative staph & $2(11.7)$ \\
Culture Negative & $4(23.5)$ \\
\hline
\end{tabular}

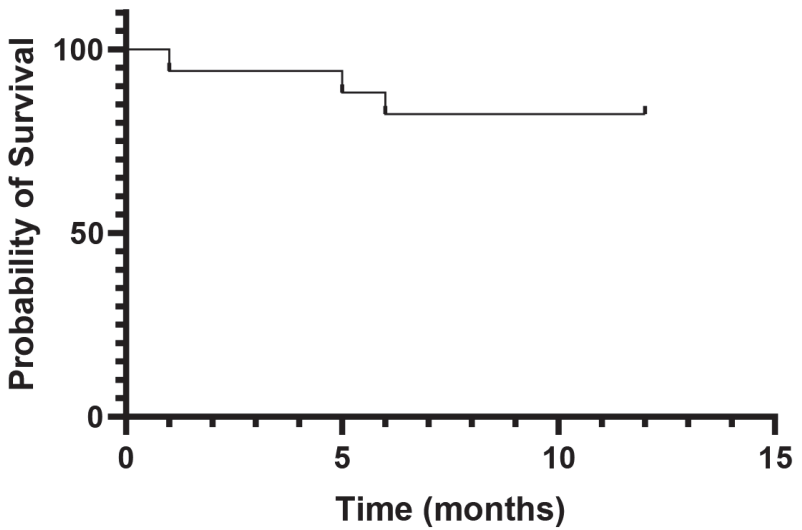

\begin{tabular}{|c|c|c|c|c|c|}
\hline Time (months) & 0 & 3 & 6 & 9 & 12 \\
\hline Number at risk & 17 & 16 & 14 & 14 & 14 \\
\hline
\end{tabular}

Survival probability estimate at one year is $0.82(95 \% \mathrm{Cl}=0.55-0.95)$

Abstract 21 Figure 1 Survival curve at 1 year for IE post-TAVI

were performed to assess the culprit microorganism and treatments for IE.

Results A total of 707 patients underwent TAVI during the study period. During a mean follow-up of 2.3 years, 17 (2.4\%) patients with IE were identified. According to the modified Duke criteria, IE was defined as definite and probable in $6(35 \%)$ and $11(65 \%)$ cases, respectively. The mean age was $78.7( \pm 13.7)$ years and $12(71 \%)$ were male. The median time from TAVI to presentation with IE was 7 months (IQR 5-13). The dominant culprit microorganisms identified were streptococci $(41.1 \%)$, with a low frequency of enterococcus faecalis $(17.6 \%)$ (table 1). Among the 17 patients with IE, there was one (6\%) major stroke and two patients (12\%) required surgical aortic valve replacement. There was one death during the initial hospitalization for IE. The KaplanMeier estimate of survival at 1 year was 0.82 (95\% CI = 0.55-0.95) (figure 1).

Conclusions This Irish TAVI cohort exhibited a similar incidence of IE post-TAVI and similar time to first presentation compared to prior international registries. There was a much lower rate of enterococcus compared to staphylococci and streptococci as the culprit microorganism. The 1-year mortality rate in this series was much lower than previously reported. 